Man and Nature

L'homme et la nature

\title{
Du Moraliste Classique Au Moraliste Des Lumières ou La Naissance Des Sciences Humaines
}

\section{H. Mydlarski}

Volume 5, 1986

URI : https://id.erudit.org/iderudit/1011857ar

DOI : https://doi.org/10.7202/1011857ar

Aller au sommaire du numéro

Éditeur(s)

Canadian Society for Eighteenth-Century Studies / Société canadienne d'étude du dix-huitième siècle

ISSN

0824-3298 (imprimé)

1927-8810 (numérique)

Découvrir la revue

Citer cet article

Mydlarski, H. (1986). Du Moraliste Classique Au Moraliste Des Lumières ou La Naissance Des Sciences Humaines. Man and Nature / L'homme et la nature, 5, 131-139. https://doi.org/10.7202/1011857ar

Copyright (c) Canadian Society for Eighteenth-Century Studies / Sociéte canadienne d'étude du dix-huitième siècle, 1986
Ce document est protégé par la loi sur le droit d'auteur. L'utilisation des services d'Érudit (y compris la reproduction) est assujettie à sa politique d'utilisation que vous pouvez consulter en ligne.

https://apropos.erudit.org/fr/usagers/politique-dutilisation/ 


\section{Du Moraliste Classique Au Moraliste Des Lumières ou La Naissance Des Sciences Humaines}

Qui dit 'moralistes' dit, selon une tradition des lettres françaises vieille de deux cents ans, un genre particulier, encore qu'assez mal défini. Un genre qui a été illustré par des auteurs tels que Pascal, La Rochefoucauld, La

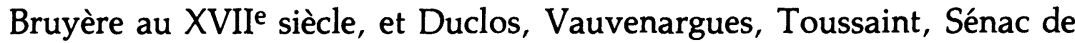
Meilhan, Chamfort, Rivarol, etc. au XVIII ${ }^{e}$. C'est dire que le terme de moraliste sera pris ici dans une acception limitée et dans le respect d'une appellation qui, pour 'tardive' qu'elle apparaisse dans l'histoire littéraire de la France, n'en renvoie pas moins, quant à ses débuts, aux grands moments de l'âge classique. A la vérité, $j$ 'espère, dans cette communication, faire d'une pierre deux coups et restituer ainsi le patrimoine sémantique d'un mot trop souvent galvaudé, 'frappé d'inflation, ${ }^{1}$ suivant l'expression d'un critique contemporain.

Ces précisions apportées, ou plutôt rappelée cette pratique née après les Lumières pour sanctionner, avec un vocable qui remontait déjà à 1690, la légitimité d'un genre en quelque sorte surnuméraire, une question se pose: pourquoi la vision collective de l'homme chez les moralistes des Lumières est-elle si différente de celle des moralistes du siècle classique? et, en corollaire, quelles conséquences, si conséquences il y a, a-telle pu entraîner? Car il ne s'agit pas de soutenir ici, comme on s'est plu à le soutenir récemment, que les moralistes du XVIII ${ }^{e}$ siècle se réclament d'une même conception de l'être que les moralistes du règne de Louis XIV; 
ou encore que leur entreprise demeure, comme chez leurs aînés, 'tributaire de la morale, de la religion et, le plus souvent, de cette "morale chrétienne" qui opère la synthèse des deux. ${ }^{2}$ Encore moins s'agit-il de prétendre qu'ils 'sont nettement en retrait sur Montaigne, en ce qui concerne 'l'autonomie,' l'affranchissement de leur pensée par rapport aux normes communément acceptées. ${ }^{3}$ Car, s'il en était ainsi, on s'expliquerait mal pourquoi Duclos avait été, dès 1745 , l'objet de la plus grave censure pour son Histoire de Louis XI, histoire où il avait fait paraître 'plusieurs endroits contraires, non seulement aux droits de la couronne sur différentes provinces du royaume, mais au respect avec lequel on doit parler de ce qui regarde la religion ou les règles des moeurs, et la conduite des principaux ministres de l'église. ${ }^{\prime}{ }^{4}$ On comprendrait aussi difficilement pourquoi Les Mours de Toussaint avaient été condamnées au feu en 1749 , quelques mois à peine après leur parution, obligeant leur auteur à aller chercher refuge en Belgique. Et que dire de Vauvenargues qui, fort de l'hétérodoxie de sa pensée, avait préféré prendre les devants de toute interdiction en déclarant haut et clair en 1746: 'J'avertis ceux qui liront ces Réflexions que, s'il y en a quelqu'une qui présente un sens peu conforme à la piété, l'Auteur désavoue ce mauvais sens, et souscrit le premier à la critique qu'on en pourra faire. ${ }^{5}$ Pour qui connaissait l'auteur, il y avait, dans un tel avertissement, de quoi rire: derrière son apparente soumission au dogme l'ancien capitaine au Régiment du Roi offrait, et Voltaire lui-même en avait déjà fait les frais, un côté assez rude, intraitable et qui ne souffrait guère la contradiction, d'où qu'elle fût venue; bref, il n'était point homme à recevoir la morale, mais à la donner. Volonté donc, parfois farouche, d'être d'abord soi-même et qui, au surplus, a distingué dans leur grande majorité les moralistes du XVIIIe siècle, les seuls qui comptent en tout cas. Tellement, par exemple, qu'aux corrections exigées par l'arrêt du Conseil lors de la condamnation de son livre Duclos avait passé outre avec un splendide dédain et que c'est encore avec dédain qu'il avait répondu à la dénonciation lancée contre lui à l'Académie Française.

Les quelques événements auxquels il vient d'être fait allusion eurent tous lieu, faut-il le rappeler, pendant la première moitié du XVIIIe siècle. C'est dire que très tôt les moralistes des Lumières eurent à coeur et, d'une certaine manière, 'à conscience' de parler en 'hommes libres'b comme le notera à plaisir plus tard Chamfort. Ce faisant, ils se mirent à propager des idées nouvelles.

De toutes les missions qu'ils attribuaient au progrès, il privilégiaient comme la plus noble et la plus exaltante celle qui ne cessait de se découvrir à eux sous la forme d'une enquête universelle et foncièrement morale sur l'homme. D'où les titres connus (et qui permettent, en partie, de les démarquer des autres écrivains): Considérations sur les moeurs de 
ce siècle, Introduction à la connaissance de l'esprit humain, Les Mours, Considérations sur l'Esprit et les Mours, Maximes et pensées, De l'homme intellectuel et moral, etc... Toutefois, comme partie intellectuellement partie souci d'objectivité, ils refusaient d'hypostasier un être idéal antérieur à l'être que nous sommes; comme ils s'interdisaient de faire contraster notre vie avec une autre vie qui, ne se mesurant pas, se refermait sur un absolu imaginaire du temps et d'une nature; bref, comme ils s'efforçaient de garder un point de vue fondé en raison, donc tout laïque, qui devait seul, à leurs yeux, investir une étude véritable de l'homme, presque au même moment il se virent en rupture avec l'Eglise. Ils ne pouvaient récuser aucun argument d'autorité ni définir l'être au monde selon des principes profanes sans, pour l'époque, dénoncer du même coup toute Loi ou toute Norme qui se posait comme extra-sociale. Pour mieux les asseoir dans un rapport de nécessité relationnelle, ils dégrevaient donc l'homme et la société d'une origine et d'un sens qui leur étaient extérieurs et étrangers. En d'autres termes, ils se tenaient à hauteur du vécu humain à dessein d'en déloger ce qu'y avaient mis les moralistes du siècle précédent: le souvenir du Péché originel et de la Révélation. Davantage: ils désacralisaient, suivant le vocabulaire de Bossuet, 'les ordres secrets de la divine Providence,' ces ordres qui continuaient à faire du royaume de France une théocratie. Ils pouvaient ainsi jeter les bases d'une enquête sur l'homme avec d'autant plus de rigueur qu'ils s'affranchissaient d'une vision christique de son 'moi' et d'une conception apostolique du monde qui les avaient en quelque sorte emmurés. La sécularisation de leur pensée devenait garante de sa scientificité. Ce qui explique pourquoi Duclos aimait tellement à rappeler que l'esprit philosophique' était 'cette supériorité de raison qui nous fait rapporter chaque chose à ses principes propres et naturels, indépendamment de l'opinion qu'en ont eue les autres hommes' (Duclos I,civ). Mais il y avait mieux. Rivarol, en qui le conservatisme réactionnaire et une vague théorie de la liberté conduisent une critique hâtive à voir un pilier du christianisme, répétait à qui voulait l'entendre que '[Dieu] est toujours absent de l'ordre moral'7 et que les moralistes d'hier, en décriant les passions, souillaient la nature. Qui plus est, il ajoutait que la religion se situait, non pas au coeur du royaume de Dieu mais bien aux confins de la terre des hommes.

Par là s'explique que, si les moralistes classiques peuvent être considérés comme des psychologues avant la lettre, les moralistes des Lumières, en revanche, doivent être regardés comme les premiers qui aient imprimé à leur recherche un caractère volontairement scientifique, au sens où se prend aujourd'hui ce mot. Leur attitude suffit seule à faire mesurer le chemin parcouru depuis la génération de Pascal qui postulait, 
à côté des sciences de raisonnement où l'expérience et la raison mènent à la connaissance, des sciences d'autorité où toute vérité est rapportée aux Saintes Ecritures. Dans l'introduction à ses Considérations sur les moeurs de ce siècle Duclos va même jusqu'à exiger que 'la science des mœurs' soit abordée à l'instar de ces autres sciences qui ' $n$ 'ont fait de vrais progrès que depuis qu'on travaille, par l'expérience, l'examen et la confrontation des faits, à éclaircir, détruire ou confirmer les systèmes' (Duclos, I, 5). Mais dès lors que l'homme n'est plus perçu a priori comme déchu ou coupable; dès lors qu'il ne figure plus comme 'le convalescent éternel chez qui le bien et le mal s'affrontent en successifs débats ${ }^{18}$ selon le mot de Saulnier; dès lors enfin qu'il n'apparaît plus comme tiraillé entre la chair et l'esprit, le fini et l'infini, la concupiscence et la pureté, force est de conclure que le mal, si mal il y a, ne peut venir que de la société. Aussi, très tôt, avec Duclos déjà, les moralistes des Lumières se prennent-ils à considérer l'homme moins en tant qu'expression de la nature humaine que dans ses rapports avec cette société. Même Vauvenargues qui est, de son siècle, le seul à en faire l'objet quasi exclusif de sa recherche, constate dès 1740 qu'il est impossible de le comprendre sans le restituer dans 'un effort de compréhension, de conciliation, d'union' 9 au milieu qui se l'approprie. Mieux encore: devant l'ampleur de la tâche à accomplir il se voit amené à circonscrire des plans d'étude, des aires d'investigation. ${ }^{10}$ Lui qui avait prétendu que 'les grandes âmes' constituaient un champ unitaire où se miroitait l'univers, le voilà maintenant astreint à inverser le sens de son rêve de connaissance 'instantanée' et à amorcer le passage d'une vision globale à une vision analytique. 'Il n'y a aucune science, finit-il par admettre, qui ne soit, à elle seule, plus vaste que l'esprit humain' (Vauvenargues, I, 399). Et il se met à ébaucher une première taxinomie humaine pour rendre les connexions que l'individu entretient avec autrui et les choses. En fait, il ne se propose rien moins que d'inaugurer le règne, non des sciences dites 'exactes' ou 'pures' (comme la physique ou les mathématiques), qu'il abhorre tout en reconnaissant leur valeur, mais des sciences 'humaines,' qu'il appelle 'utiles,' faute d'un certain langage (Vauvenargues, II, 72) ${ }^{11}$ mais aussi parce que le mot, en tant qu'apodictique du vrai, s'entend alors 'comme un principe interne, essentiel à l'ordre et à la cohérence des sociétés civilisées. ${ }^{12}$ Dès qu'à ses yeux est admis le principe d'une relation objective entre les phénomènes sociaux comme il en existe une entre les phénomènes physiques, il n'est plus en effet de recherche qui ne se voie appelée, au seuil même de sa démarche, à être inscrite dans une systématique du savoir. Or, comme il ne lui paraît pas douteux que l'homme se reçoive d'une dépendance forcée de la matière et de la société, au besoin d'introduire un peu d'ordre dans le matériau et le processus discursif de la réflexion correspond chez lui la nécessité de 
postuler, comme composantes de son être, les rapports et les liens qui le nouent à son milieu. Vauvenargues tire même, avant Quesnay et plus intelligemment que lui, tellement sa construction est ample et équilibrée, les grandes lignes d'une économie politique. Aux suggestions qu'il faudrait apporter des solutions radicales aux problèmes de la nation malade, il répond par exemple:

On a peut-être des moyens moins violents: rendre les avantages du commerce supérieurs aux dommages du luxe; avilir l'oisiveté, et protéger l'industrie, l'agriculture et la population; empêcher, autant qu'il se peut, la disproportion trop grande des fortunes; simplifier la perception des revenus du Roi; en un mot, bien d'autres ressources, qu'une connaissance plus profonde de l'état de chaque province pourrait révéler aux ministres; car je crois qu'il est impossible, dans la condition présente du Royaume, de faire quelque bien, autrement que dans le détail, et de trouver, par exemple, de ces moyens simples, qui opèrent par une impulsion unique et universelle. La plupart de ces beaux systèmes n'ont qu'un endroit de réel, et ne sont pas proportionnés à leur fin: l'Etat est comme une balance; un poids trop fort emporterait d'un coup l'équilibre. (Vauvenargues II, 69)

Les physiocrates ont-ils jamais rien écrit de plus sensé? Les moralistes des Lumières sont par ailleurs persuadés qu'il y a abus (et injustice) à fonder sur quelque science que ce soit des espoirs de préhension de l'homme total. Que toute investigation sérieuse débouche sui generis sur un pluridisciplinaire, loin de les confirmer dans un sentiment d'infaillibilité, leur rappelle que nous sommes tous uniques et aussi fuyants que les aspirations profondes de notre 'moi.' Dans un texte particulièrement drôle par son faux-sérieux, Sénac de Meilhan se plaît même à imaginer le jour où la science aura parfaitement élucidé la nature de l'homme. Ce jour-là, écrit-il, tout sera réduit 'en axiomes, en maximes constantes' (noblesse oblige!) et 'l'homme ... exposé aux yeux de tous sera comme une pendule à jour dont on voit tous les ressorts, dont l'oeil suit tous les mouvements. ${ }^{13}$ Quoi qu'il en soit, ajoute le pince sans-rire, on aura 'des thermomètres sûrs' (Sénac, 62) qui indiqueront son genre d'esprit et en fixeront le degré. Et comme, à la limite, dans dix ou douze générations, il finira par tomber en léthargie dans un monde vide et insipide qui n'alimentera plus sa soif de connaître, peut-être n'aura-t-on d'autre moyen, pour le sortir de sa torpeur moderne, que celui $d^{\prime \prime} u n$ déluge qui replonge tout dans l'ignorance' (Sénac, 65)!

Mais ne nous y trompons pas. Cette satire de la science dans un domaine dont elle avait été écartée par les moralistes classiques ne prouve pas qu'elle ait été condamnée au XVIII siècle par les auteurs qui nous occupent ici. Bien au contraire. Ce que le texte de Sénac de Meilhan atteste, 
c'est la réalité d'exigences nouvelles, qui s'inscrivent dans la marche même du progrès mais qu'il importe d'apprécier à leur juste valeur si on ne veut pas se couvrir de ridicule. Les moralistes des Lumières sont en fait si entiers, disons mieux, se veulent si entiers dans leur application du démonstratif à la recherche, qu'ils estiment rédhibitoire toute histoire qui n'offrirait pas une documentation sûre quant aux faits et logique quant à leur enchaînement. En ce sens, ils sont encore parmi les premiers à poser des critères objectifs pour juger du passé et lui donner un éclairage séculier. Il y a plus. lls refusent de prêter à la société une malléabilité factice et, dans leur vision d'une humanité en constante élaboration, ils apparaissent comme les tenants originaires du déterminisme historique. 'La révolution de 1789 est le résultat d'un assemblage de causes agissant depuis des siècles,' pose Chamfort comme en exergue à ses Tableaux historiques de la Révolution française (Chamfort II, 159). Et dans ses Pensées il constate qu' 'il y a, dans tout, une maturité qu'il faut attendre' et que 'les hommes les plus extraordinaires' n'ont jamais été responsables à eux seuls des changements de fond, qu' ils ont été secondés par les circonstances les plus favorables, et par l'esprit de leur temps' (Chamfort l, 446). Même Sénac de Meilhan, dans l'Emigré par surcroît, ne fait pas que regarder la Révolution, malgré ses horreurs, comme l'aboutissement quasi inéluctable d'une société et d'un régime, encore la voit-il porteuse d'un règne de justice et de tolérance à l'empreinte d'un lendemain. Et que dire de Duclos qui, bien des années avant 1789 , se sent déjà emporté par les événements et espère voir s'établir de son vivant, après des siècles de maturation et d'épreuves, un ordre nouveau et plus juste? Seul peut-être Rivarol nie la possibilité d'une évolution historique. Persuadé que jamais rien ne saura tirer le peuple de la barbarie au sceau de laquelle, à l'en croire, celui-ci est irrémédiablement marqué, il ne cherche qu'à maintenir les Français, par toutes sortes de règles et de craintes, dans une totale soumission. Ce qui ne l'empêche pas d'épiloguer sur les causes de la Révolution dans ses Extraits du Journal politique et national et de se demander par quels vices ou par quelles aberrations la constitution, le fonctionnement et la conduite du gouvernement, l'administration publique, la nature des institutions, les critères du régime, etc ... ont pu produire, dans un pays qui paraissait ancré dans les plus anciennes lois et les plus vieilles traditions, des événements qui ont bouleversé le cours de son histoire. 'Car on ne peut se détacher de cette intéressante discussion', remarque-t-il encore, postulant par là une nouvelle problématique (Rivarol IV, 232). Mais c'est justement cette discussion, cette problématique, née autant de la Révolution que des Lumières (les Lumières n'ont jamais voulu la Révolution), qui, ensemble avec d'autres, jettera les premiers fondements des sciences politiques. La complexité des questions 
soulevées; le scrupule avec lequel chaque détail, même le plus anodin d'apparence, est intégré au tissu conjonctif du corps politique; les retombées présentes de décrets passés des siècles plus tôt; l'analyse des différentes classes sociales, de leur esprit et de leurs aspirations; les indélébiles préjugés de certains groupes d'hommes; la sottise des uns et l'avarice crétinisante des autres; la conscience d'une évolution des mentalités; la situation économique du royaume; les salaires; les périodes de vaches maigres et de vaches grasses; les moyens de distribution du stock alimentaire; bref, tout ou presque, même le hasard, devient objet d'étude pour les moralistes. Malgré l'absence d'outils statistiques que Vauvenargues avait, au reste, déjà déplorée! Qu'ils soient engagés politiquement ne saurait non plus enlever à leur mérite qu'à celui de nos propres savants qui n'hésitent guère à tomber, parfois dogmatiquement, dans la profession de foi. Et c'est ainsi que Rivarol, par exemple, dresse le bilan d'une réflexion qui, pour s'être exercée avec rigueur sur les données d'une science riche mais en son premier essor, n'en reflète pas moins une prise de position politique simpliste et forcée, voire outrageusement réactionnaire:

On a voulu faire de la France, écrit-il ironiquement, une grande loterie où chacun pût gagner sans y mettre. Parcourez la série des décrets de l'Assemblée, vous croirez entendre, à l'éloquence près, la voix des Gracques et de tous ces tribuns qui adulaient la canaille latine, et qui finirent par renverser la République. L'Assemblée nationale, en détruisant la hiérarchie des conditions, si conforme à la nature des monarchies, pense obtenir un meilleur ordre des choses: penserait-elle aussi, en donnant aux notes la même valeur, et en les rangeant toutes sur une même ligne, créer d'autres accords et donner au monde une nouvelle harmonie? (Rivarol IV, 168)

Hommes de lettres se transformant en hommes de sciences parce qu'ils refusent de se laisser dépasser par des ébranlements qui leur échappent, les pionniers de l'histoire économique et sociale sont aussi, à l'occasion, les devanciers lointains des linguistes d'aujourd'hui. Au moins pressentent-ils que les civilisations se reçoivent des mains de la langue qu'elles parlent. Au moins entrevoient-ils qu'il n'est guère de société qui puisse véritablement se comprendre, de moment de l'humanité qui puisse être légitimement rendu, si n'est prise en considération la nature même de l'expression qui investit l'une et l'autre. L'erreur n'a donc pas été forcément gratuite qui a consisté à donner pour titre De la nature du langage en général, au lieu de De l'homme intellectuel et moral, au premier volume des œuvres complètes de Rivarol (1808). 'Une langue, écrit celuici, doit être considérée sous le même point de vue que la société qui la 
parle, puisqu'il est vrai que les mots sont dans l'une ce que les hommes sont dans l'autre' (Rivarol l, xv). La connaissance des lois de l'humanité se fonde à ce point dans celle des lois du langage qu'après son Discours sur l'universalité de la langue française où il s'essaie à poser les jalons de l'histoire du français et d'un peuple, Rivarol songe déjà à entreprendre un dictionnaire de conception rigoureusement moderne. Passons sur son parti-pris: il est d'époque; et il ne nous incombe pas de jeter la pierre à l'homme parce qu'il se fait fort d'expliquer pourquoi la langue française est universelle en son siècle, mais peut-être à l'Académie de Berlin qui en propose le sujet. Toujours est-il que la pensée de Rivarol se veut résolument scientifique, quelque humeur qu'elle fasse paraitre, et qu'elle se révèle comme une approche nouvelle qui incitera à la réflexion une Europe non seulement littéraire et mondaine mais encore savante et ... littéralement séduite. De la même veine on notera aussi les intelligentes Remarques sur la Grammaire générale et raisonnée ${ }^{14}$ de Duclos.

Que conclure? A mesure que se sécularise la pensée des moralistes des Lumières, l'étude de l'homme s'objective et énonce les critères de recherche et de méthode qui fonderont les sciences humaines. Tant que la notion de créature déchue avait prévalu, le comportement de l'individu, au fond, pour les moralistes chrétiens du siècle classique, n'avait ni posé de problèmes ni demandé à être mieux compris. Le dogme du péché originel avait suffi seul à en rendre compte et à l'inscrire dans un principe du mal dont il ne pouvait sortir que par la médiation christique. D'où l'ironie cinglante et, plus tard, le ton indigné avec lesquels Pascal avait condamné les casuistes de son temps qui déculpabilisaient ses 'intentions' comme s'il avait pu avoir par lui-même d'autres intentions que mauvaises. D'où encore les limites du champ réflexif qui l'excluaient de son univers social, économique et politique, et dans lesquelles s'était enfermé, malgré son originalité, La Rochefoucauld. Mais avec l'éveil de l'esprit philosophique les attitudes changent. Les moralistes plaident pour des points de vue laïques dans tous les domaines de la recherche et soutiennent qu'il faut prendre la nature pour ce qu'elle est, en dehors de tout a priori métaphysique ou moral de ce qu'elle devrait être. La nature, à leurs yeux, est, tout simplement, et ne pourrait pas ne pas être ce qu'elle est. Et dans son fait d'être elle a en partage l'homme qui s'associe à elle pour exister et à qui il serait aussi absurde d'imputer à vice son 'moi' qu'à l'arbre son feuillage. Aussi, face à l'éternel problème du mal, préfèrent-ils observer ce 'moi' dans les rapports qu'il entretient avec la société, soit pour lui faire chercher 'ses avantages personnels dans le plan du bien général' (Duclos 1, 20) soit pour l'obliger à inventer un ordre nouveau d'où serait bannie l'injustice. Par là ils amorcent le tournant décisif qui orientera désormais leur discours du côté de la politique, et s'apprêtent à 
marquer les hautes heures de la pensée et de l'éloquence révolutionnaires et contre-révolutionnaires.

\section{H. MYDLARSKI \\ University of Calgary}

\section{Notes}

1 Louis Van Delft, 'La spécificité du moraliste classique', Revue d'histoire littéraire de la France, No. 4 (juillet/août 1980), 542. Voir aussi, du même auteur, Le moraliste classique - essai de définition et de typologie (Genève: Droz 1982), p. 13.

2 Louis Van Delft, 'La réflexion sur le moraliste', Romanistische Zeitschrift für Literaturgeschichte, H.J. (1980), p. 85.

3 Ibid., p. 85.

4 Duclos, Oeuvres complètes, éd. M. Auger, 9 vols. (Paris: Janet et Cotelle 1820-1821), I, xj. Ci-après abrégé: Duclos I, dans le texte.

5 Vauvenargues, Oeuvres et Oeuvres posthumes et œuvres inédites, éd. D.-L. Gilbert, 2 vols. (Paris: Furne et Cie 1857), I, 373. Ci-après abrégé: Vauvenargues I ou II, dans le texte.

6 C'est ainsi, par exemple, que Chamfort qualifie Duclos. Voir Chamfort, Oeuvres complètes, éd. P.R. Auguis, 5 vols. (Paris: Chaumerot Jeune 1824-1825), III, 296. Ci-après abrégé: Chamfort I, II ou III, dans le texte.

7 Rivarol, Oeuvres complètes, 5 vols. (Paris: Fayolle et Chênedollé 1808), I, 320. Ci-après abrégé: Rivarol I ou IV, dans le texte.

8 V.-L. Saulnier, La littérature française du siècle classique, 7e éd. (Paris: P.U.F. 1963), p. 61.

9 Georges Poulet, Etudes sur le temps humain: II. La distance intérieure (Paris: Plon 1952), p. 55.

10 Voir notre 'Vauvenargues, ou le moraliste devant la nécessité d'une économie politique', Transactions of the Fifth International Congress on the Enlightenment, II, Section 8: The beginnings and development of political economy (Oxford: The Voltaire Foundation 1980), cxci, 820

11 Voir aussi ibid., 820.

12 Bernard Plongeron, 'Echec à la sécularisation des Lumières: la religion comme lien social,' Transactions of the Sixth International Congress on the Enlightenment (Oxford: The Taylor Foundation 1983), 68

13 Sénac de Meilhan, Considérations sur l'esprit et les mœurs, éd. F. Caussy (Paris: E. Sansot 1905), 61. Ci-après abrégé: Sénac, dans le texte.

14 Grammaire dite 'de Port-Royal.' 